\title{
Nonlinear multidimensional gravity and the Australian dipole
}

\author{
K.A. Bronnikov ${ }^{a, b, 1}$, V.N. Melnikov ${ }^{a, b, 2}$, S.G. Rubin ${ }^{c, 3}$, and I.V. Svadkovsky ${ }^{c, 4}$ \\ a Center for Gravitation and Fundamental Metrology, VNIIMS, 46 Ozyornaya St., Moscow 119361, Russia \\ $b$ Institute of Gravitation and Cosmology, PFUR, 6 Miklukho-Maklaya St., Moscow 117198, Russia \\ c National Research Nuclear University "MEPhI", Kashirskoe sh. 31, Moscow, 115409 Russia
}

\begin{abstract}
The existing observational data on possible variations of fundamental physical constants (FPC) confirm more or less confidently only a variability of the fine structure constant $\alpha$ in space and time. A model construction method is described, where variations of $\alpha$ and other FPCs (including the gravitational constant $G$ ) follow from the dynamics of extra space-time dimensions in the framework of curvature-nonlinear multidimensional theories of gravity. An advantage of this method is a unified approach to variations of different FPCs. A particular model explaining the observable variations of $\alpha$ in space and time has been constructed. It comprises a FRW cosmology with accelerated expansion, perturbed due to slightly inhomogeneous initial data.
\end{abstract}

\section{Introduction}

The problem of possible variations of the fundamental physical constants (FPC) in time and space is one of the most challenging problems of modern physics, directly related to the central problem of unification of all interations. It traces back to Dirac's and Eddington's famous papers of the 1930s and since then gains much attention in both theoretical and experimental studies.

However, to date, a variability of only one FPC has been revealed by observations more or less confidently, it is the fine structure constant $\alpha$. The analysis of absorption spectra of various ions in the radiation of distant quasars, performed in the recent years (above all, from the data obtained at the Keck telescope on the Hawayian islands), has led to a conclusion that $\alpha$ is changing with time, so that in the past it was slightly smaller than now (the relative change $\delta \alpha / \alpha$ is about $10^{-5}[1]$ ). In 2010, an analysis of new data obtained at the VLT (Very Large Telescope), located in Chile, and their comparison with the Keck data led to a conclusion on spatial variations of $\alpha$, i.e., on its dependence on the direction of observations. The VLT observations in the Southern part of the celestial sphere gave values of the parameter $\alpha$ in the past slightly larger than now. This anisotropy has a dipole nature [2,3] and has been termed "the Australian dipole" [4]. The dipole axis is located at a declination of $-61 \pm 9^{\circ}$ and at a right ascention of $17.3 \pm 0,6$ hours. The deflection of $\alpha$ value at an arbitrary point $r$ of space from its modern value $\alpha_{0}$, measured on Earth, is

$$
\delta \alpha / \alpha_{0}=(1.10 \pm 0.25) \times 10^{-6} r \cos \psi,
$$

where $\psi$ is the angle between the direction of observation and the dipole axis, while the distance $r$ is measured in billions of light years. The confidence level of this result (as compared with a

\footnotetext{
${ }^{1}$ e-mail: kb20@yandex.ru

2e-mail: melnikov@phys.msu.ru

${ }^{3}$ e-mail: sergeirubin@list.ru

${ }^{4}$ e-mail: igor_svadkovsky@mail.ru
} 
"monopole" model where values of $\alpha$ are the same in all directions) has been estimated as $4.1 \sigma$. A more detailed discussion of the observational data can be found, e.g., in [3].

Let us also mention the laboratory experimental data on possible FPC variations in the modern epoch. The tightest constraints on $\alpha$ variations have been obtained by comparison of readings of atomic clocks using optical transitions in $\mathrm{Al}$ and $\mathrm{Hg}$ ions (without using cesium clocks that have become classic) [5]: $(d \alpha / d t) / \alpha=(-1.6 \pm 2.3) \times 10^{-17}$ per year. This result is of the same order of magnitude as the tightest constraints obtained previously from an isotopic composition analysis of the decay products in the natural nuclear reactor that operated in the Oklo region (Gabon) about 2 billion years ago. Unlike the laboratory data, the Oklo results [6] and, in particular, the tightest constraint $[7,8]$

$$
-3.7 \times 10^{-17} / \mathrm{yr}<d(\ln \alpha) / d t<3.1 \times 10^{-17} / \mathrm{yr}
$$

rely on the assumption that during these 2 billion years the value of $\alpha$ changed uniformly, if changed at all. This assumption looks rather natural but actually follows from nowhere.

Thus in the modern epoch, at least on Earth since the Oklo times, the parameter $\alpha$ did not change more rapidly than by approximately $10^{-17}$ per year. If, on the other hand, we use the distant quasar data and take a mean value of $d(\ln \alpha) / d t$ for about 10 billion years, we shall obtain a variation rate of about $10^{-15}$ per year. Therefore one can conclude that at times earlier (maybe much earlier) than 2 billion years ago the value of $\alpha$ changed relatively rapidly but afterwards stopped or almost stopped to change. The task of theory was to explain such a behavior; however, if one takes into account the most recent observations [2,9], one should add the necessity of exlaining the spatial variations of $\alpha$. Though, one cannot exclude the opportunity that the variations of $\alpha$ are purely spatial in nature whereas the time dependence is related to the finiteness of the velocity of light: being located at a fixed point and at fixed time, we receive signals from distant regions of the Universe emitted at earlier cosmological epochs, and it is therefore impossible to separate spatial and temporal dependences of the parameters.

Let us briefly discuss the theoretical models describing variations of $\alpha$. Thus, following the pioneering ideas of Dirac and Eddington, Dicke and Peebles [10] in 1962 considered variations of $\alpha$ in cosmological models admitting a variable gravitational interaction intensity. Staniukovich [11] in 1965 discussed different variants of combined FPC variations in connection with Dirac's Large Number Hypothesis. Bekenstein [12] in 1982 described a model of $\alpha$ variations on the basis of the most general assumptions on the electromagnetic interaction: covariance, gauge invariance, causality and invariance with respect to time reversion. This led to a modified Maxwell electrodynamics and provided a certain dynamics of $\alpha$.

Since the advent of astronomical evidence on possible time variations of $\alpha$, there emerged a whole class of new models describing such variations by introducing certain scalar fields. Thus, Sandvik et al. [13] proposed a cosmological extension of Bekenstein's theory [12] with a term of the form $-\frac{1}{4} F_{\mu \nu} F^{\mu \nu} e^{-2 \psi}$ in the initial Lagrangian, where the scalar field $\psi$ interacts only with the electromagnetic field $F^{\mu \nu}$. The effect of the field $\psi$ in the dynamics of the expanding Universe was also considered. It was shown that in this model $\alpha$ remained almost constant in the radiationdominated epoch, slightly increased in the matter-dominated epoch and approaches a constant value at times when the Universe expansion accelerates due to the presence of a positive cosmological constant.

Spatial variations of $\alpha$ were also discussed much before they were claimed to be really discovered, in attempts to explain the discrepancy between cosmological and terrestrial data on $\alpha(t)[14,15]$.

In the recent attempts to explain both temporal and spatial variations of the fine structure constant, quite popular are models assuming the existence of domain walls connected with scalar 
field dynamics (see, e.g., $[16,17])$. Thus, in [17] the initial action contains a dilaton-like scalar field $\phi$ interacting with the electromagnetic field and having a potential of the form $V(\phi)=\frac{1}{4} \lambda\left(\phi^{2}-\eta^{2}\right)^{2}$. A domain wall is formed due to spontaneous symmetry breakdown. At points separated by the domain wall the values of $\alpha$ are different, which can explaing the observable variations if the wall intersects our Hubble volume.

In [18] it has been shown that in $F(R)$ gravity it is possible to obtain a static solution in the form of an effective (gravitational) domain wall, and that the choice of a logarithmic nonminimal interaction of the electromagnetic field with gravity in the form

$$
-\frac{1}{4}\left[1+\ln \left(\frac{R}{R_{0}}\right)\right] F_{\mu \nu} F^{\mu \nu}
$$

(where $R_{0}$ is the modern value of the scalar curvature) makes it possible to describe variations of $\alpha$, whose value grows as the curvature $R$ decreases.

Olive et al. [19] discuss a model with two domain walls, where the scalar field potential has three minima:

$$
V(\phi)=\lambda\left(|\Phi|^{2}-\frac{\eta^{2}}{2}\right)^{2}-\sqrt{2} i \epsilon\left(\Phi^{3}-\left(\Phi^{*}\right)^{3}\right)+V_{0}
$$

It turns out that such a model much better describes the observational data than a similar one [17] with a single domain wall.

The paper [20] suggests an extension of the previous BSBM (Bekenstein-Sandvik-BarrowMagueijo) theory $([12,13])$ by introducing a dependence of the coupling constant $\omega$ of the scalar field $\psi$ on the field itself, so that the Lagrangian contains the terms $L_{\psi}=-\frac{1}{2} \omega(\psi) \partial_{\mu} \psi \partial^{\mu} \psi$ and $L_{\mathrm{em}}=-\frac{1}{4} F_{\mu \nu} F^{\mu \nu} e^{-2 \psi}$. The choice of $\omega(\psi)$ allows for obtaining both growing and falling time dependences of $\alpha$. This model differs from those with domain walls in that the variations of $\alpha$ are smooth and continuous, and a choice between these models must be easy with future more precise and reliable observational data.

Mariano and Perivolaropoulos [21] have reported on a correlation between the spatial distribution of $\alpha$ values and the dipole anisotropy of the dark energy distribution. In the same paper they have suggested a theoretical model explaining this correlation (named "extended topological quintessence") which naturally predicts inhomogeneous spherical distributions of both the dark energy and the values of $\alpha$. The model assumes the existence of a huge global monopole with a size of Hubble order, which nonminimally interacts with the electromagnetic field. There emerge mutually related distributions of different parameters with a dipole anisotropy from the viewpoint of any observer located outside the monopole center. The monopole is formed after a phase transition in a set of three scalar fields with an $\mathrm{O}(3)$ symmetric Lagrangian.

In a later paper [22] the same authors support their inferences by the data on one more anisotropy also seeming to exist and to be aligned with other "dipoles", the so-called Large-Scale Velocity Flows (Dark Flow), i.e., recent indications that there is a large-scale peculiar velocity flow with an amplitude larger than $400 \mathrm{~km} / \mathrm{s}$ on scales up to $100 h^{-1} \mathrm{Mpc}(z \leq 0.03)$.

It is also important to mention the theoretical models considering FPC variations in the framework of unification scenarios. In particular, P. Langacker et al. [23] consider possibile variations of coupling constants due to physics at very high energies, where the gauge couplings may be unified. It means that one should treat a joint variation of the fine structure and strong coupling constants.

Similarly to [23], X. Calmet and H. Fritzsch [24] discuss FPC variations in the context of Grand unification. They show that such a consideration leads to small time shifts of the nucleon mass, the magnetic moment of the nucleon and the weak coupling constant, and it is expected to have a relative change of the nucleon mass larger than that of $\alpha$ by a factor of $\sim 40$. 
A more detailed discussion of theoretical models involving unification scenarios to explain the FPC variations can be found in [25].

It should be noted that all the above approaches, to explain variations of $\alpha$, introduce scalar fields whose existence and manner of interaction with the electromagnetic field are postulated from the outset and are not explained in any way. In what follows, it will be shown how the scalar fields and their interaction law with electromagnetism naturally follow from nonlinear multidimensional gravity. Spatial variations of $\alpha$ are explained by a large-scale inhomogeneity of this scalar field. The magnitude of this inhomogeneity is constrained by CMB observations [26].

The approach we are using has been formulated in [27], where a methodology was suggested allowing for a transition from multidimensional gravity with higher derivatives to Einstein-Hilbert gravity with effective scalar fields. Later on this approach was successfully applied for a unified description of the inflationary stage of the Universe and the modern secondary inflation [28] and an explanation of the origin of the Higgs field [29]; a mechanism of cascade reduction of multidimensional space to the observable one was suggested [30,31]. It has been shown under which conditions the compact extra dimensions become stationary (i.e., have a constant volume), and the cause of their maximum symmetry was found [32].

The present study has been performed in the framework of this approach and is an example of its employment. The paper is organized as follows. Sec. 2 briefly describes the general formalism used. In this framework, in Sec. 3 we build a homogeneous and isotropic cosmological model able to describe the present accelerated Universe along with a time dependence of the fine structure constant $\alpha$. In Sec. 4 , this cosmological model is slightly perturbed on large scale, which enables us to explain spatial variations of $\alpha$. Sec. 5 is a brief conclusion.

\section{Multidimensional gravity and its reduction}

Consider a $\left(D=4+d_{1}\right)$-dimensional manifold with the metric

$$
d s^{2}=g_{\mu \nu} d x^{\mu} d x^{\nu}+\mathrm{e}^{2 \beta(x)} b_{a b} d x^{a} d x^{b}
$$

where the extra-dimensional metric components $b_{a b}$ are independent of $x^{\mu}$, the observable four space-time coordinates.

The $D$-dimensional Riemann tensor has the nonzero components

$$
\begin{aligned}
R_{\rho \sigma}^{\mu \nu} & =\bar{R}_{\rho \sigma}^{\mu \nu}, \\
R_{\nu a}^{\mu a} & =\delta_{b}^{a} B_{\nu}^{\mu}, \quad B_{\nu}^{\mu}:=\mathrm{e}^{-\beta} \nabla_{\nu}\left(\mathrm{e}^{\beta} \beta^{\mu}\right), \\
R^{a b}{ }_{c d} & =\mathrm{e}^{-2 \beta} \bar{R}_{c d}^{a b}+\delta_{c d}^{a b} \beta_{\mu} \beta^{\mu},
\end{aligned}
$$

where capital Latin indices cover all $D$ coordinates, the bar marks quantities obtained from $g_{\mu \nu}$ and $b_{a b}$ taken separately, $\beta_{\mu} \equiv \partial_{\mu} \beta$ and $\delta^{a b}{ }_{c d} \equiv \delta_{c}^{a} \delta_{d}^{b}-\delta_{d}^{a} \delta_{c}^{b}$. The nonzero components of the Ricci tensor and the scalar curvature are

$$
\begin{aligned}
R_{\mu}^{\nu} & =\bar{R}_{\mu}^{\nu}+d_{1} B_{\mu}^{\nu} \\
R_{a}^{b} & =\mathrm{e}^{-2 \beta} \bar{R}_{a}^{b}+\delta_{a}^{b}\left[\square \beta+d_{1}(\partial \beta)^{2}\right], \\
R & =\bar{R}[g]+\mathrm{e}^{-2 \beta} \bar{R}[b]+2 d_{1} \square \beta+d_{1}\left(d_{1}+1\right)(\partial \beta)^{2},
\end{aligned}
$$

where $(\partial \beta)^{2} \equiv \beta_{\mu} \beta^{\mu}, \square=\nabla^{\mu} \nabla_{\mu}$ is the d'Alembert operator while $\bar{R}[g]$ and $\bar{R}[b]$ are the Ricci scalars corresponding to $g_{\mu \nu}$ and $b_{a b}$, respectively. Let us also present, using similar notations, the 
expressions for two more curvature invariants, the Ricci tensor squared and the Kretschmann scalar $\mathcal{K}=R^{A B C D} R_{A B C D}$ :

$$
\begin{gathered}
R_{A B} R^{A B}=\bar{R}_{\mu \nu} \bar{R}^{\mu \nu}+2 d_{1} \bar{R}_{\mu \nu} B^{\mu \nu}+d_{1}^{2} B_{\mu \nu} B^{\mu \nu}+\mathrm{e}^{-4 \beta} \bar{R}_{a b} \bar{R}^{a b} \\
+2 \mathrm{e}^{-2 \beta} \bar{R}[b]\left[\square \beta+d_{1}(\partial \beta)^{2}\right]+d_{1}\left[\square \beta+d_{1}(\partial \beta)^{2}\right]^{2}, \\
\mathcal{K}=\overline{\mathcal{K}}[g]+4 d_{1} B_{\mu \nu} B^{\mu \nu}+\mathrm{e}^{-4 \beta} \overline{\mathcal{K}}[b]+4 \mathrm{e}^{-2 \beta} \bar{R}[b](\partial \beta)^{2}+2 d_{1}\left(d_{1}-1\right)\left[(\partial \beta)^{2}\right]^{2} .
\end{gathered}
$$

Suppose now that $b_{a b}$ describes a compact $d_{1}$-dimensional space of nonzero constant curvature, i.e., a sphere $(K=1)$ or a compact $d_{1}$-dimensional hyperbolic space $(K=-1)$ with a fixed curvature radius $r_{0}$ normalized to the $D$-dimensional analogue $m_{D}$ of the Planck mass, i.e., $r_{0}=$ $1 / m_{D}$ (we use the natural units, with the speed of light $c$ and Planck's constant $\hbar$ equal to unity). We have

$$
\begin{aligned}
\bar{R}_{c d}^{a b} & =K m_{D}^{2} \delta_{c d}^{a b}, \\
\bar{R}_{a}^{b} & =K m_{D}^{2}\left(d_{1}-1\right) \delta_{a}^{b}, \\
\bar{R}[b] & =K m_{D}^{2} d_{1}\left(d_{1}-1\right)=R_{b} .
\end{aligned}
$$

The scale factor $b(x) \equiv \mathrm{e}^{\beta}$ in (3) is thus kept dimensionless; $R_{b}$ has the meaning of a characteristic curvature scale of the extra dimensions.

Consider, in the above geometry, a sufficiently general curvature-nonlinear theory of gravity with the action

$$
\begin{aligned}
S & =\frac{1}{2} m_{D}^{D-2} \int \sqrt{{ }^{D} g} d^{D} x\left(L_{g}+L_{m}\right), \\
L_{g} & =F(R)+c_{1} R^{A B} R_{A B}+c_{2} \mathcal{K},
\end{aligned}
$$

where $F(R)$ is an arbitrary smooth function, $c_{1}$ and $c_{2}$ are constants, $L_{m}$ is a matter Lagrangian and ${ }^{D} g=\left|\operatorname{det}\left(g_{M N}\right)\right|$.

The extra coordinates are easily integrated out, reducing the action to four dimensions:

$$
S=\frac{1}{2} \mathcal{V}\left[d_{1}\right] m_{D}^{2} \int \sqrt{{ }^{4} g} d^{4} x \mathrm{e}^{d_{1} \beta}\left[L_{g}+L_{m}\right]
$$

where ${ }^{4} g=\left|\operatorname{det}\left(g_{\mu \nu}\right)\right|$ and $\mathcal{V}\left[d_{1}\right]$ is the volume of a compact $d_{1}$-dimensional space of unit curvature.

Eq. (10) describes a curvature-nonlinear theory with non-minimal coupling between the effective scalar field $\beta$ and the curvature. Let us simplify it in the following way (putting, for convenience, $m_{D}=1$, so that all quantities are now expressed in ( $D$-dimensional) Planck units:

(a) Express everything in terms of $4 \mathrm{D}$ variables and $\beta(x)$; we have, in particular,

$$
R=R_{4}+\phi+f_{1}, \quad R_{4}=\bar{R}[g], \quad f_{1}=2 d_{1} \square \beta+d_{1}\left(d_{1}+1\right)(\partial \beta)^{2},
$$

where we have introduced the effective scalar field

$$
\phi(x)=R_{b} \mathrm{e}^{-2 \beta(x)}=K d_{1}\left(d_{1}-1\right) \mathrm{e}^{-2 \beta(x)}
$$

The sign of $\phi$ coincides with $k= \pm 1$, the sign of curvature in the $d_{1}$ extra dimensions.

(b) Suppose that all quantities are slowly varying, i.e., consider each derivative $\partial_{\mu}$ (including those in the definition of $\bar{R}$ ) as an expression containing a small parameter $\varepsilon$; neglect all quantities of orders higher than $O\left(\varepsilon^{2}\right)$ (see $\left.[27,31]\right)$. 
(c) Perform a conformal mapping leading to the Einstein conformal frame, where the 4-curvature appears to be minimally coupled to the scalar $\phi$.

In the decomposition (11), both terms $f_{1}$ and $R_{4}$ are regarded small in our approach, which actually means that all quantities, including the 4D curvature, are small as compared with the $D$-dimensional Planck scale. The only term which is not small is $\phi$, and we can use a Taylor decomposition of the function $F(R)=F\left(\phi+R_{4}+f_{1}\right)$ :

$$
F(R)=F\left(\phi+R_{4}+f_{1}\right) \simeq F(\phi)+F^{\prime}(\phi) \cdot\left(R_{4}+f_{1}\right)+\ldots,
$$

with $F^{\prime}(\phi) \equiv d F / d \phi$. Substituting this, and the corresponding decompositions of the expressions (6) and (7), into Eq. (10), we obtain, up to $O\left(\varepsilon^{2}\right)$, the following effective gravitational Lagrangian $L_{g}$ in Eq. (10):

$$
L_{g}=F^{\prime}(\phi) R_{4}+F(\phi)+F^{\prime}(\phi) f_{1}+c_{*} \phi^{2}+2 c_{1} \phi \square \beta+2\left(c_{1} d_{1}+2 c_{2}\right)(\partial \beta)^{2}
$$

with $c_{*}=c_{1} / d_{1}+2 c_{2} /\left[d_{1}\left(d_{1}-1\right)\right]$.

The action (10) with (14) is typical of a scalar-tensor theory (STT) of gravity in a Jordan frame. To study the dynamics of the system, it is helpful to pass on to the Einstein frame. Applying the conformal mapping

$$
g_{\mu \nu} \mapsto \widetilde{g}_{\mu \nu}=|f(\phi)| g_{\mu \nu}, \quad f(\phi)=\mathrm{e}^{d_{1} \beta} F^{\prime}(\phi),
$$

after a lengthy calculation, we obtain the action in the Einstein frame as

$$
\begin{aligned}
S & =\frac{1}{2} \mathcal{V}\left[d_{1}\right] \int \sqrt{\widetilde{g}}\left(\operatorname{sign} F^{\prime}\right) L \\
L & =\widetilde{R}_{4}+K_{\mathrm{E}}(\phi)(\partial \phi)^{2}-2 V_{\mathrm{E}}(\phi)+\widetilde{L}_{m}, \\
\widetilde{L}_{m} & =\left(\operatorname{sign} F^{\prime}\right) \frac{\mathrm{e}^{-d_{1} \beta}}{F^{\prime}(\phi)^{2}} L_{m} \\
K_{\mathrm{E}}(\phi) & =\frac{1}{4 \phi^{2}}\left[6 \phi^{2}\left(\frac{F^{\prime \prime}}{F^{\prime}}\right)^{2}-2 d_{1} \phi \frac{F^{\prime \prime}}{F^{\prime}}+\frac{1}{2} d_{1}\left(d_{1}+2\right)+\frac{4\left(c_{1}+c_{2}\right) \phi}{F^{\prime}}\right], \\
-2 V_{\mathrm{E}}(\phi) & =\left(\operatorname{sign} F^{\prime}\right) \frac{\mathrm{e}^{-d_{1} \beta}}{F^{\prime}(\phi)^{2}}\left[F(\phi)+c_{*} \phi^{2}\right],
\end{aligned}
$$

where the tilde marks quantities obtained from or with $\widetilde{g}_{\mu \nu}$; the indices are raised and lowered with $\widetilde{g}_{\mu \nu}$; everywhere $F=F(\phi)$ and $F^{\prime}=d F / d \phi$; $\mathrm{e}^{\beta}$ is expressed in terms of $\phi$ using (12).

Let us consider the electromagnetic field $F_{\mu \nu}$ as matter in the initial Lagrangian, putting

$$
L_{m}=\alpha_{1}^{-1} F_{\mu \nu} F^{\mu \nu},
$$

where $\alpha_{1}$ is a constant. After reduction to four dimensions this expression acquires the factor $\mathrm{e}^{d_{1} \beta}$ arising from the metric determinant: $\sqrt{D^{D}}=\sqrt{{ }^{4} g} \mathrm{e}^{d_{1} \beta}$. In the subsequent transition to the Einstein picture the expression $\sqrt{{ }^{4} g} F_{\mu \nu} F^{\mu \nu}$ remains the same (it is the well-known conformal invariance of the electromagnetic field), hence the Lagrangian (17) takes the form

$$
\widetilde{L}_{m}=\alpha_{1}^{-1} \mathrm{e}^{d_{1} \beta} F_{\mu \nu} F^{\mu \nu},
$$

and for the effective fine structure constant $\alpha$ we obtain

$$
\frac{\alpha}{\alpha_{0}}=\mathrm{e}^{d_{1}\left(\beta_{0}-\beta\right)}
$$

where $\alpha_{0}$ and $\beta_{0}$ are values of the respective quantities at a fixed space-time point, for instance, where and when the observation is taking place. 


\section{The cosmological model}

Depending on the choice of $F(R)$, the parameter $c_{1}$ and $c_{2}$ and the matter Lagrangian in the action (9), the theory under consideration can lead to a great variety of cosmological models. Some of them were discussed in [27], mostly those related to minima of the effective potential (19) at nonzero values of $\phi$. Such minima correspond to stationary states of the scalar $\phi$ and consequently of the scale factor $b=\mathrm{e}^{\beta}$ of the extra dimensions. If the minimum value of the potential is positive, it can play the role of a cosmological constant that launches an accelerated expansion of the Universe.

Here, we would like to focus on another minimum of the potential $V_{\text {Ein }}$, existing for generic choices of the function $F(R)$ with $F^{\prime}>0$ and located at the point $\phi=0$. The asymptotic $\phi \rightarrow 0$ corresponds to growing rather than stabilized extra dimensions: $b=\mathrm{e}^{\beta} \sim 1 / \sqrt{|\phi|} \rightarrow \infty$. A model with such an asymptotic growth at late times may still be of interest if the growth is sufficiently slow and the size $b$ does not reach detectable values by now. Let us recall that the admissible range of such growth comprises as many as 16 orders of magnitudes if the $D$-dimensional Planck length $1 / m_{D}$ coincides with the $4 \mathrm{D}$ one, i.e., about $10^{-33} \mathrm{~cm}$ : the upper bound corresponds to lengths about $10^{-17} \mathrm{~cm}$ or energies of the order of a few $\mathrm{TeV}$. This estimate certainly changes if there is no such coincidence.

One should note that small values of $\phi$ to be considered here are still very large as compared to $4 \mathrm{D}$ quantities, and so our general assumptions are well justified. Indeed, according to (12),

$$
|\phi|=\frac{d_{1}\left(d_{1}-1\right)}{b^{2}}
$$

where $b \lesssim 10^{16}$, hence $|\phi| \gtrsim d_{1}^{2} \cdot 10^{-32}$, while the quantity $\widetilde{R}_{4}$, if identified with the curvature of the modern Universe, is of the order $10^{-122}$ in Planck units (that is, close to the Hubble parameter squared, or (the Hubble time) ${ }^{-2}$, see also Eq. (34) below).

Let us check whether it is possible to describe the modern state of the Universe by an asymptotic form of the solution for $\phi \rightarrow 0$ as a spatially flat cosmology with the 4D Einstein-frame metric

$$
d \widetilde{s}_{4}^{2}=d t^{2}-a^{2}(t) d \vec{x}^{2}
$$

where $a(t)$ is the Einstein-frame scale factor. We shall be are working in the framework of quadratic gravity with a cosmological constant, i.e. ${ }^{5}$

$$
F(\phi)=-2 \Lambda_{D}+F_{2} \phi^{2}
$$

where $\Lambda_{D}$ is the initial cosmological constant. Then, substituting $F^{\prime}=2 \phi$ and $F^{\prime \prime}=2$, we obtain for the kinetic and potential terms in the Lagrangian (16) in the first approximation in $\phi$ :

$$
\begin{aligned}
& K_{\mathrm{E}} \approx K_{0} /\left(2 \phi^{2}\right), \quad K_{0}=\frac{1}{2}\left[\frac{1}{2} d_{1}^{2}-d_{1}+6+2\left(c_{1}+c_{2}\right)\right] ; \\
& V_{\mathrm{E}} \approx V_{0} \mathrm{e}^{-2 \bar{d} \beta}, \quad V_{0}=\frac{\Lambda_{D}}{4 d_{1}^{2}\left(d_{1}-1\right)^{2}}, \quad 2 \bar{d}=d_{1}-4 .
\end{aligned}
$$

It is clear that this model can work only if $d_{1}>4$. In terms of $\beta$ instead of $\phi$, the Lagrangian takes the form

$$
L=\widetilde{R}_{4}+2 K_{0}(\partial \beta)^{2}-2 V_{0} \mathrm{e}^{-2 \bar{d} \beta}+\widetilde{L}_{m},
$$

\footnotetext{
${ }^{5}$ We assume for certainty $\phi>0$, or, which is the same according to (12), $K=+1$, but everything can be easily reformulated for $\phi<0$.
} 
Neglecting the gravitational influence of the electromagnetic field (that is, considering only vacuum models), one can write down the independent components of the Einstein and scalar field equations with the unknowns $\beta(t)$ and $a(t)$ in the form

$$
\begin{aligned}
3 \frac{\dot{a}^{2}}{a^{2}} & =K_{0} \dot{\beta}^{2}+V_{0} \mathrm{e}^{-2 \bar{d} \beta}, \\
\ddot{\beta}+3 \frac{\dot{a}}{a} \dot{\beta} & =\frac{V_{0} \bar{d}}{K_{0}} \mathrm{e}^{-2 \bar{d} \beta} .
\end{aligned}
$$

These equations, corresponding to a scalar field with an exponential potential, can be solved exactly but the solution looks rather involved, and for our purpose more preferable is the comparatively simple approximate solution that can be obtained in the slow-rolling approximation; the latter should be acceptable at late times. Let us suppose that

$$
|\ddot{\beta}| \ll 3 \frac{\dot{a}}{a} \dot{\beta}, \quad K_{0} \dot{\beta}^{2} \ll V_{0} \mathrm{e}^{-2 \bar{d} \beta},
$$

and neglect the corresponding terms in Eqs. (27) and (28). Then, expressing the quantity $\dot{a} / a$ from (27) and substituting it into (28), we obtain

$$
\dot{\beta}=\frac{\bar{d} \sqrt{V_{0}}}{K_{0} \sqrt{3}} \mathrm{e}^{-\bar{d} \beta},
$$

whence

$$
\mathrm{e}^{\bar{d} \beta}=\frac{\bar{d}^{2}}{K_{0}} \sqrt{\frac{V_{0}}{3}}\left(t+t_{1}\right),
$$

where $t_{1}$ is an integration constant. For the scale factor $a(t)$ we have

$$
\frac{\dot{a}}{a}=\frac{p}{t+t_{1}} \quad \Rightarrow \quad a=a_{1}\left(t+t_{1}\right)^{p}, \quad a_{1}=\text { const }, \quad p=\frac{K_{0}}{\bar{d}^{2}} .
$$

Substituting the solution to the slow-rolling conditions (29), we make sure that they hold as long as $p \gg 1$, or in terms of the input parameters of the theory,

$$
p=\frac{d_{1}^{2}-2 d_{1}+12+4\left(c_{1}+c_{2}\right)}{\left(d_{1}-4\right)^{2}} \gg 1 .
$$

We will assume that this condition holds.

A further interpretation of the results depends on which conformal frame is regarded physical (observational) [33,34], and this in turn depends on the manner in which fermions appear in the (so far unknown) underlying unification theory involving all interactions.

Let us adopt the simplest hypothesis that the observational picture coincides with the Einstein picture and make some estimates. Thus, the inverse of the modern value of the Hubble parameter (the Hubble time) is estimated as

$$
t_{H}=1 / H_{0}=a_{0} / \dot{a}_{0} \approx 4,4 \times 10^{17} \mathrm{c} \approx 8 \times 10^{60} t_{\mathrm{pl}},
$$

where $t_{\mathrm{pl}}$ is the Planck time and the index "0" marks quantities belonging to the present time, which is a usual notation in cosmology. From (32) it follows that $H_{0}=p /\left(t_{0}+t_{1}\right)$, whence

$$
t_{*}:=t_{0}+t_{1}=p t_{H} \gg t_{H}
$$


With $p \gg 1$, the model satisfies the observational constraints on the factor $w$ in the effective equation of state $p=w \rho$ of dark energy that causes the accelerated expansion of the Universe: at $w=$ const we have $a \sim t^{2 /(3+3 w)}$, consequently, $w=-1+2 /(3 p)$ is a number close to -1 : for example, to have $w \approx-0.99$, one should put only $p=66$. Meanwhile, the recent observational data allow for a comparatively large range of $w$ [35-38] but anyway admitting $w=-1$ corresponding to a cosmological constant. This follows from combining the recent measurements of cosmic microwave background anisotropies, Supernovae luminosity distances, baryonic acoustic oscillations, and $H(z)$ measurements, though different tests lead to different confidence intervals.

Furthermore, the "internal" scale factor $b(t)=\mathrm{e}^{\beta}$ grows much slower than $a(t)$ :

$$
b(t)=b_{0}\left(\frac{t+t_{1}}{t_{*}}\right)^{1 / \bar{d}}, \quad b_{0}=\left(\frac{1}{H_{0}} \sqrt{\frac{V_{0}}{3}}\right)^{1 / \bar{d}} .
$$

Using the expression for $V_{0}$ from (25), one can estimate the initial parameter $\Lambda_{D}$, connecting it with the size of the extra factor space $b_{0}$ : in Planck units,

$$
\Lambda_{D}=12 H_{0}^{2} d_{1}^{2}\left(d_{1}-1\right)^{2} b_{0}^{d_{1}-4} \approx \frac{3}{16} d_{1}^{2}\left(d_{1}-1\right)^{2} b_{0}^{d_{1}-4} \times 10^{-120} .
$$

As already mentioned, the "internal" scale factor $b=\mathrm{e}^{\beta}$ should be in the range $1 \ll b_{0} \lesssim 10^{16}$ in Planck units. The estimate (37) shows that the present model makes much easier the wellknown "cosmological constant problem" (the difficulty of explaining why in standard cosmology $\Lambda_{\text {standard }} \sim 10^{-122}$ in Planck units). For instance, if (in the admissible range) $b_{0}=10^{15}$ and $d_{1}=12$, it follows $\Lambda_{D}=3267$ without any indication of fine tuning.

Let us estimate the possible range of the parameters $c_{1}$ and $c_{2}$ in the action (9). The present model describes only the modern stage of the Universe evolution, but it should admit an improvement after which it will account for other stages, including the early inflation. Then one should require that the curvature-nonlinear terms in the initial Lagrangian should not violate our slowchange approximation, see Sec. 2 This leads to the condition $c_{1,2} \ll 10^{11}$. Indeed, during inflation, the Hubble parameter is $H \sim 10^{-6}$ in Planck units, while the scalar curvature at inflation, when the 4D geometry is approximately de Sitter, is estimated as $R \simeq 12 H^{2} \sim 10^{-11}$. Assuming that the Ricci and Riemann tensor components have the same order of magnitude, we find that the condition $R \gg c_{1} R^{A B} R_{A B}$, used above in the framework of the slow-change approximation, will be violated if $c_{1}$ is too large. The upper bound of the parameter $c_{2}$ is obtained in a similar way.

The smallness of the observed variations of $\alpha$ leads to another constraint on $c_{1}$ and $c_{2}$ : according to $(38)$,

$$
\alpha / \alpha_{0}=\left(b / b_{0}\right)^{-d_{1}}=\left(\frac{t+t_{1}}{t_{0}+t_{1}}\right)^{-2 d_{1} /\left(d_{1}-4\right)} \approx 1-\frac{2 d_{1}}{d_{1}-4} \frac{t-t_{0}}{t_{*}}
$$

so that $\dot{\alpha} / \alpha \sim 10^{-10} / p$ per year. By the empirical data, this quantity cannot be larger than about $10^{-17}$ per year. A comparison leads to the constraint $p \gtrsim 10^{7}$ and hence the effective equation-ofstate parameter $w$ is equal to -1 up to seven meaningful digits. Taking into account the relation (33) between $p$ and the input parameters $c_{1}$ and $c_{2}$, we obtain similar bounds on these parameters if the number of extra dimensions $d_{1}$ is not too large.

Thus the allowed range of $c_{1}$ and $c_{2}$ (assuming that they are of the same order of magnitude),

$$
10^{7} \lesssim c_{1,2} \ll 10^{11}
$$

is wide enough, which means that any fine tuning is absent. 
One of the well-known constraints on Kaluza-Klein-like cosmologies is the requirement of a sufficiently slow evolution of the internal scale factor, e.g., according to [39], the extra-dimensional volume should not have changed by more than $10 \%$ since the times of primordial nucleosynthesis. This requirement rests on the relation between the effective Newtonian gravitational constant $G_{\mathrm{N}}$ and the volume of extra dimensions. However, in the Einstein conformal frame used here, $G_{\mathrm{N}}=$ const by definition, therefore the above constraint does not apply here. Still, even if we passed on to the Jordan frame, the relative variations of $G_{\mathrm{N}}$ would be the same as those of $\alpha$ and obey the law (38), i.e., within about $10^{-5}$ for the Hubble time.

In the next section we shall see that the inequality $p \gtrsim 10^{7}$ and consequently $c_{1,2} \gtrsim 10^{7}$ are substantially relaxed in the perturbed model.

\section{Spatial variations of $\alpha$}

In the previous section we discussed the properties of a homogeneous model which does not contain any spatial variation of $\alpha$ (and any other physical quantity). Let us try to describe variations of $\alpha$ by taking into account spatial perturbations of the scalar field and the metric. Only long-wave perturbations will be of interest for us, with characteristic lengths of the order of the horizon size.

An observed statistically isotropic sky means that there is no preferred axis. Nevertheless, super-horizon components were produced by quantum fluctuations at the beginning of inflation in the same way as fluctuations of smaller scale. It means that the dipole component must exist though hardly observed due to its contamination by the Doppler effect caused by the motion of our Local Group with respect to the CMB. Hence there must exist a weakly expressed distinguished direction along which the metric and scalar field inhomogeneity is most clearly pronounced.

Accordingly, we now choose a metric more general than (23),

$$
d s_{\mathrm{E}}^{2}=\mathrm{e}^{2 \delta \gamma} d t^{2}-a(t)^{2} \mathrm{e}^{2 \delta \lambda} d x^{2}-a(t)^{2} \mathrm{e}^{2 \delta \eta}\left(d y^{2}+d z^{2}\right),
$$

where $x$ is the distinguished direction and $\delta \gamma, \delta \lambda, \delta \eta \ll 1$ are functions of $x$ and $t$. In addition, we replace the effective scalar field $\beta(t)$ with $\beta(t)+\delta \beta(x, t)$.

Then the relevant Einstein-scalar equations corresponding to the Lagrangian (26) can be written as follows (preserving only terms linear in the "deltas"):

$$
\begin{aligned}
& \delta \ddot{\beta}+\frac{3 \dot{a}}{a} \delta \dot{\beta}+\dot{\beta}(\delta \dot{\lambda}-\delta \dot{\gamma})-\frac{1}{a^{2}} \delta \beta^{\prime \prime}+\frac{1}{2 K_{0}} \delta\left(V_{\beta} \mathrm{e}^{2 \gamma}\right)=0, \\
& \frac{\dot{a}}{a}(\delta \dot{\lambda}-\delta \dot{\gamma})=\delta\left(V \mathrm{e}^{2 \gamma}\right), \\
& \frac{\dot{a}}{a} \delta \gamma^{\prime}=K_{0} \dot{\beta} \delta \beta^{\prime}
\end{aligned}
$$

where we have chosen the gauge (in other words, the reference frame in perturbed space-time) $\delta \eta \equiv 0$, the dot and the prime stand for $\partial / \partial t$ and $\partial / \partial x$, respectively. We have also denoted $V=V_{\mathrm{E}}=V_{0} \mathrm{e}^{-2 \bar{d} \beta}$ and $V_{\beta}=d V / d \beta$.

Integration of (43), without loss of generality, leads to

$$
\delta \gamma=\frac{K_{0}}{H} \dot{\beta} \delta \beta
$$

where, as before, $H=\dot{a} / a$. This equation enables us to estimate the quantity $\delta \beta$. Indeed, according to the CMB data [26], we can take $\delta \gamma \sim 10^{-5}$, while the coefficient before $\delta \beta$ is of the order of 
unity at the present epoch (according to Eq. (32) we have $\left.\left(K_{0} / H_{0}\right) \dot{\beta}\left(t=t_{0}\right)=K_{0} /(\bar{d} \cdot p)=\bar{d} \sim 1\right)$, we obtain $\delta \beta \sim 10^{-5}$.

Substituting this $\delta \gamma$ to (41) and taking the difference $\delta \dot{\lambda}-\delta \dot{\gamma}$ from (42), we finally arrive at the following single wave equation for $\delta \beta$ :

$$
\delta \ddot{\beta}+\frac{3 \dot{a}}{a} \delta \dot{\beta}-\frac{1}{a^{2}} \delta \beta^{\prime \prime}+\delta \beta\left[\frac{2 \dot{\beta}^{2}}{H^{2}} V K_{0}+\frac{2 \dot{\beta}}{H} V_{\beta}+\frac{1}{2 K_{0}} V_{\beta \beta}\right]=0 .
$$

with an arbitrary constant $K_{0}$ and an arbitrary potential $V(\beta)$. In our case, with $V=V_{0} \mathrm{e}^{-2 \bar{d} \beta}$ and $K_{0}$ given in (25), we obtain

$$
\delta \ddot{\beta}+\frac{3 \dot{a}}{a} \delta \dot{\beta}-\frac{1}{a^{2}} \delta \beta^{\prime \prime}+\frac{2 V_{0} \mathrm{e}^{-2 \bar{d} \beta}}{p} \delta \beta=0,
$$

while the background quantities $a(t)$ and $\beta(t)$ are determined by the solution (31), (32). It remains to find a solution for $\delta \beta$ which, being added to the background $\beta(t)$, would be able to account for the observed picture of variations of $\alpha$.

Since the background is $x$-independent, we can separate the variables and assume

$$
\delta \beta=y(t) \sin k\left(x+x_{0}\right)
$$

where $k$ has the meaning of a wave number, of order of the cosmological horizon scale, and $y(t)$ must be as small as $10^{-5}$. Then $y(t)$ obeys the equation

$$
\ddot{y}+\frac{3 p}{t+t_{1}} \dot{y}+\left[\frac{k^{2}}{a_{1}^{2}\left(t+t_{1}\right)^{2 p}}+\frac{6 p}{\left(t+t_{1}\right)^{2}}\right] y=0 .
$$

Since the equation (47) has been derived in a certain approximation and describes only a restricted period of time close to the present epoch, it is reasonable to seek the solution in the form of a Taylor series:

$$
y(t)=y_{0}+y_{1}\left(t-t_{0}\right)+\frac{1}{2} y_{2}\left(t-t_{0}\right)^{2}+\ldots, \quad y_{i}=\text { const. }
$$

Then $y_{0}$ and $y_{1}$ can be fixed at will as initial conditions, and Eq. (47) leads to expressions of $y_{2}, y_{3}, \ldots$ in terms of $y_{0}$ and $y_{1}$. Even more than that, for a certain neighborhood of $t=t_{0}$ we can simply suppose $y=y_{0}+y_{1}\left(t-t_{0}\right)$. Actually, this approximation is good enough for $t-t_{0} \ll t_{*}=t_{0}-t_{1}$.

In this approximation we obtain the following expression for variations of $\alpha$ :

$$
\frac{\alpha}{\alpha_{0}} \approx 1-\frac{d_{1}}{\bar{d}} \frac{t-t_{0}}{t_{*}}-d_{1} \sin \left[k\left(x+x_{0}\right)\right]\left[y_{0}+y_{1}\left(t-t_{0}\right)\right]+O\left(\epsilon^{2}\right)
$$

where $O\left(\epsilon^{2}\right)$ means $O\left(\left(t-t_{0}\right)^{2} / t_{*}^{2}\right)$. Assuming that the observer is located at $x=0$ and requiring $\alpha / \alpha_{0}=1+O\left(\epsilon^{2}\right)$ at $x=0$, we obtain the condition

$$
y_{1} \sin \left(k x_{0}\right)=-1 /\left(\bar{d} t_{*}\right) .
$$

This explains very small, if any, variations of $\alpha$ on Earth at present and since the Oklo times. Indeed, since $2 \times 10^{9} \mathrm{yr} \approx \frac{1}{7} t_{H}$ while $t_{*}=p t_{H}$, the addition $O\left(\epsilon^{2}\right)$ is of the order of $1 /\left(50 p^{2}\right)$, where $p \gg 1$. If we take, for instance, $p=1000$, then at the Oklo time $\left(2 \times 10^{9}\right.$ years ago $)$ we obtain a relative $\alpha$ variation of the order $0.5 \times 10^{-8}$, which makes about $0.25 \times 10^{-17}$ per year. 
A substitution of (50) and (50) into (49) at $t-t_{0}=-x$ for $x>0$ gives

$$
\alpha / \alpha_{0} \approx 1-d_{1} y_{0} \sin \left(k x_{0}\right)+d_{1} y_{0} k x \cos \left(k x_{0}\right)+O\left(\epsilon^{2}\right)
$$

at $x \ll t_{*}$. The same result is obtained if we substitute $t-t_{0}=x$ for $x<0$.

Fig. 1 compares the observational data and the predictions of our model with the parameters indicated there. Recall that we are considering long-wave fluctuations, such that $k \leq 1 / r_{H} \sim 0.1$ (billion years) ${ }^{-1}$ (where $r_{H}$ is the modern horizon size), with a small magnitude $y_{0} \leq 10^{-5}$. The relations obtained are in good agreement with these estimates. We are using the conventional normalization $a_{0}=1$.

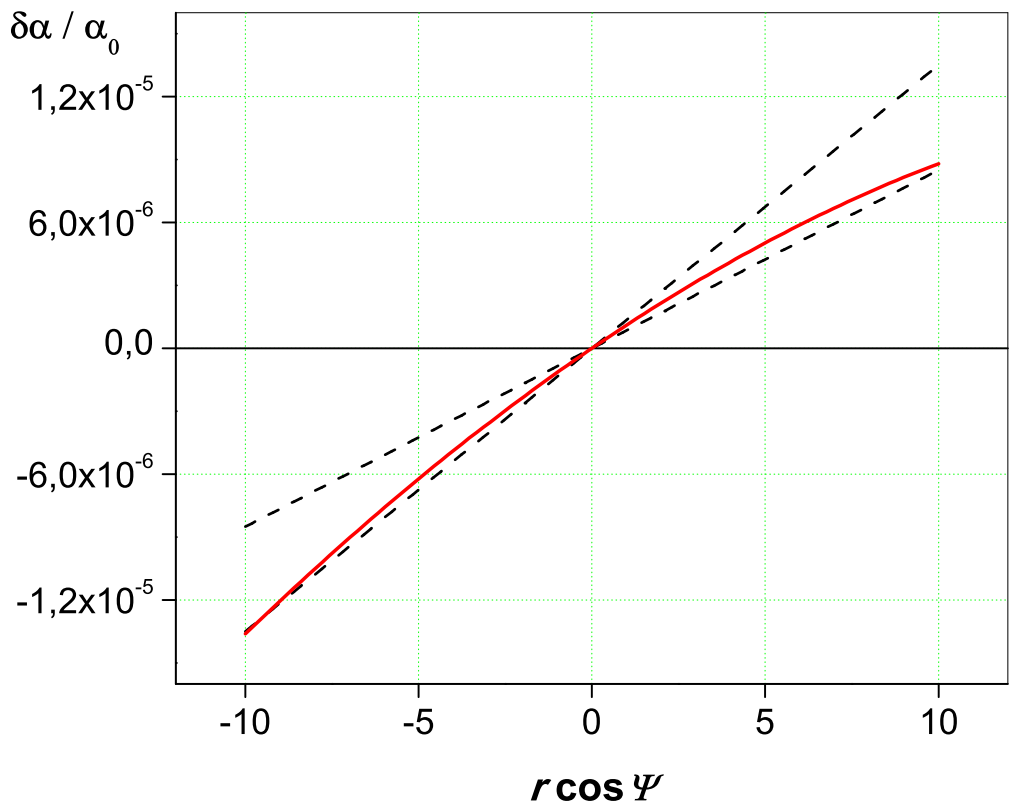

Figure 1: The $r$ dependence of $\delta \alpha / \alpha_{0}$ (the distance $r$ is measured in billions of light years). The dashed lines correspond to Eq. (1), the solid red line to Eq. (49) at the parameter values $d_{1}=12$, $p=10^{7}, y_{0}=-4.7 \times 10^{-6}, y_{1}=-10^{-7}$ (bill. years) ${ }^{-1}, k=0.02$ (bill. light years) ${ }^{-1}, x_{0}=1$ billion of light years.

Evidently, our model, in addition to the input theoretical parameters like $d_{1}, c_{1}, c_{2}$, contains the parameters $k, x_{0}, y_{0}, y_{1}$, depending on the initial form of the extra space metric.

In the framework of chaotic inflation, these parameters vary in different regions of the visible part of the Universe. Their choice enables us to explain the spatial variations of $\alpha$ in agreement with the observations [2]. Actually, there are only two conditions imposed on them: (50) and the relationship identifying (51) with the expression (1) at $r=x$ and $\cos \psi=1$, i.e., on the dipole axis. We obtain (in Planck units)

$$
d_{1} y_{0} k \cos \left(k x_{0}\right) \approx-2 \times 10^{-66} .
$$

(This numerical value is used for obtaining the solid line in Fig. 1.) The small constant shift of the $\alpha$ value at $x=0$ against the background does not change the interpretation of the results obtained. It should be stressed that (52) is not a fine-tuning relation but simply fitting of the model parameters to the observational data. In fact, the very small number in the r.h.s. of (52) results 
from the natural scale of $k \sim 1 / r_{H}$, where $r_{H} \sim 10^{28} \mathrm{~cm} \sim 10^{61} \ell_{\mathrm{pl}}$ is the Hubble radius in terms of the Planck length $\ell_{\mathrm{pl}}$; five more orders of magnitude in (52) are related to the smallness of $\alpha$ variations.

The input parameters $c_{1}$ and $c_{2}$ are now not so strongly constrained by the condition of slow variations of $\alpha$ on Earth: this condition is already provided by the equality (50) if we take $p \gtrsim 1000$, hence $c_{1}+c_{2} \gtrsim 1000$. The approximation $p \gg 1$, in which our solution has been obtained, then also holds quite well. The inequality (39) is thus replaced by a much weaker one:

$$
1000 \lesssim c_{1} \sim c_{2} \ll 10^{11}
$$

\section{Conclusion}

We have studied the possible effect of extra dimensions on large-scale variations of the fine structure constant $\alpha$ in space and time. In the multidimensional paradigm under consideration, the observable values of $\alpha$ and probably other physical quantities, including fundamental constants, depend on the size of the extra factor space. Variations of the dark energy density can be mentioned as an example. Indeed, the space-time variations of the energy density are dominated by those of the potential $V=V_{\mathrm{E}}$ given in (25). The relative variation $\delta V / V=-2 \bar{d} \delta \beta$ is of the same order of magnitude as the space-time variations of $\alpha$ according to (38). They are too small to be observed in the near future.

We have discussed the dipole component only, but it seems evident that the same basis is applicable to higher multipoles in $\alpha$ variations. It means that the observational data, being quite uncertain, "feel" these components, and further observations may detect them.

We have focused on the behavior of $\alpha$ because it is the only fundamental constant for which there are more or less reliable data indicating its variations. We are also planning to analyze the behavior of other constants, above all, the gravitational constant and the particle masses.

The model described here does not consistently include other kinds of matter than dark energy (represented by a scalar field of multidimensional origin). However, even such a simple model shows an agreement with the observational data (see Fig. 1). The same numerical parameters also well agree with the CMB constraints which impose an upper bound on the fluctuation magnitude of the extra-dimensional metric.

An advantage of the present model of $\alpha$ variation against many others (e.g., $[16,17,19])$ is that it assumes a common origin of dark energy and FPC variations. Our model also predicts the existence of higher multipoles in $\alpha$ variations.

\section{Acknowledgments}

The authors wish to thank A. Panov for his interest in our work. The work of S.R. and I.S. was supported in part by the Ministry of Education and Science of the Russian Federation, project 14.A18.21.0789. 


\section{References}

[1] J.K. Webb et al., Further evidence for cosmological evolution of the fine structure constant. Phys. Rev. Lett. 87, 091301 (2001).

[2] J.K. Webb et al., Evidence for spatial variation of the fine structure constant. Phys. Rev. Lett. 107, 191101 (2011); ArXiv: 1008.3907.

[3] Julian A. King et al., Spatial variation in the fine-structure constant - new results from VLT/UVES, ArXiv: 1202.4758.

[4] J.C. Berengut and V.V. Flambaum, Astronomical and laboratory searches for space-time variation of fundamental constants. J. Phys. Conf. Ser. 264, 012010 (2011); Arxiv: 1009.3693.

[5] T. Rosenband et al., Observation of the $1 \mathrm{~S}_{¿}$ ¿ $3 \mathrm{P} 0$ Clock Transition in ${ }^{27} \mathrm{Al}^{+}$. Phys. Rev. Lett. 98, 220801 (2007).

[6] A.I. Shlyakhter, Direct test of the constancy of fundamental nuclear constants. Nature 260, 340 (1976).

[7] Yu.V. Petrov et al., Natural nuclear reactor Oklo and variation of fundamental constants. Part 1: Computation of neutronics of fresh core. Phys. Rev. C 74, 064610 (2006); ArXiv: hep-ph/0506186.

[8] C.R. Gould et al., Time-variability of alpha from realistic models of Oklo reactors. Phys.Rev. C74, 024607 (2006); ArXiv: nucl-ex/0701019.

[9] T. Chiba. The constancy of the constants of Nature: Updates. Prog. Theor. Phys. 126, 993-1019 (2011); ArXiv: 1111.0092.

[10] R.H. Dicke and P.J.E. Peebles, Phys. Rev. 128, 2006 (1962).

[11] K.P. Staniukovich, The Gravitational Field and Elementary Particles, Nauka, Moscow, 1965 (in Russian).

[12] J.D. Bekenstein, Phys. Rev. D 25, 1527 (1982).

[13] H.B. Sandvik, J.D. Barrow, J. Magueijo, Phys. Rev. Lett. 88, 031302 (2002); astro-ph/0107512.

[14] D.F. Mota and J.D. Barrow, Varying alpha in a more realistic universe. Phys. Lett. B 581, 141 (2004); astro-ph/0306047.

[15] D.F. Mota and J.D. Barrow, Local and global variations of the fine structure constant. Mon. Not. Roy. Astron. Soc. 349, 291 (2004); astro-ph/0309273.

[16] T. Chiba and M. Yamaguchi, Runaway domain wall and space-time varying $\alpha$. JCAP 1103, 044 (2011); ArXiv: 1102.0105.

[17] K.A. Olive, M. Peloso, J.-P. Uzan, The wall of fundamental constants. Phys. Rev. D 83, 043509 (2011); ArXiv: 1011.1504.

[18] K. Bamba, S. Nojiri, S.D. Odintsov, ArXiv: 1107.2538.

[19] K.A. Olive, M. Peloso, A.J. Peterson, Where are the walls? ArXiv: 1204.4391.

[20] J.D. Barrow and S.Z.W. Lip, A generalized theory of varying alpha. ArXiv: 1110.3120.

[21] A. Mariano and L. Perivolaropoulos, Is there correlation between fine structure and dark energy cosmic dipoles? ArXiv: 1206.4055. 
[22] A. Mariano and L. Perivolaropoulos, CMB maximum temperature asymmetry axis: alignment with other cosmic asymmetries. ArXiv: 1211.5915.

[23] P. Langacker, G. Segre, M.J. Strassler, implications of gauge unification for time variation of the fine structure constant. Phys. Lett. B 528, 121-128 (2002); ArXiv: hep-ph/0112233.

[24] X. Calmet, H. Fritzsch, The cosmological evolution of the nucleon mass and the electroweak coupling constants. Eur. Phys. J. C 24, 639-642 (2002); ArXiv: hep-ph/0112110.

[25] J.-P. Uzan, Varying constants, gravitation and cosmology. Living Reviews in Relativity, vol. 14, no. 2; ArXiv: 1009.5514.

[26] N. Jarosik et al., Seven-year Wilkinson Microwave Anisotropy Probe (WMAP) observations: sky maps, systematic errors, and basic results. FREE ISSUE: Astrophys. J. Suppl. 192, 14 (2011).

[27] K.A. Bronnikov and S.G. Rubin, Self-stabilization of extra dimensions. Phys. Rev. D 73, 124019 (2006).

[28] K.A. Bronnikov, S.G. Rubin, and I.V. Svadkovsky, Multidimensional world, inflation and modern acceleration Phys. Rev. D 81, 084010 (2010).

[29] S.V. Bolokhov, K.A. Bronnikov, and S.G. Rubin, Extra dimensions as a source of the electroweak model, Phys. Rev. D 84, 044015 (2011).

[30] S.G. Rubin and A.S. Zinger, The Universe formation by a space reduction cascade with random initial parameters, Gen. Rel. Grav. 44, 2283 (2012); ArXiv: 1101.1274.

[31] K.A. Bronnikov and S.G. Rubin, Black Holes, Cosmology and Extra Dimensions (World Scientific, Singapore, 2012).

[32] A.A. Kirillov, A.A. Korotkevich, and S.G. Rubin, Emergence of symmetries. Phys. Lett. B 718, 237240 (2012); ArXiv: 1205.1108.

[33] K.A. Bronnikov and V.N. Melnikov, On observational predictions from multidimensional gravity. Gen. Rel. Grav. 33, 1549 (2001); gr-qc/0103079.

[34] K.A. Bronnikov and V.N. Melnikov, Conformal frames and D-dimensional gravity, gr-qc/0310112, in: Proc. 18th Course of the School on Cosmology and Gravitation: The Gravitational Constant. Generalized Gravitational Theories and Experiments (30 April-10 May 2003, Erice), Ed. G.T. Gillies, V.N. Melnikov and V. de Sabbata, (Kluwer, Dordrecht/Boston/London, 2004) pp. 39-64.

[35] Planck Collaboration, Planck 2013 results. XVI. Cosmological parameters. ArXiv: 1303.5076.

[36] Najla Said, Carlo Baccigalupi, Matteo Martinelli, Alessandro Melchiorri, Alessandra Silvestri, New constraints on the dark energy equation of state. ArXiv: 1303.4353

[37] Omer Farooq, Bharat Ratra, Constraints on dark energy from the Ly $\alpha$ forest baryon acoustic oscillations measurement of the redshift 2.3 Hubble parameter. ArXiv: 1212.4264.

[38] Omer Farooq, Data Mania, Bharat Ratra, Hubble parameter measurement constraints on dark energy. ArXiv: 1211.4253.

[39] Nima Arkani-Hamed, Savas Dimopoulos, Gia Dvali, Phenomenology, astrophysics and cosmology of theories with sub-millimeter dimensions and TeV scale quantum gravity. Phys. Rev. D 59, 086004 (1999); hep-ph/9807344. 